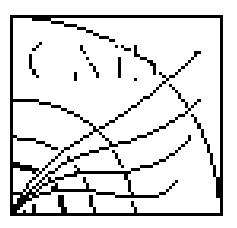

'l'he Compact Mwon Solenoid Experiment

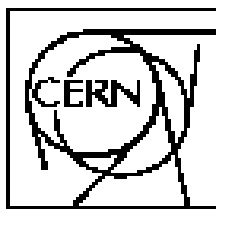

August 22, 2004

\title{
Uniformity Measurements across the Area of the CMS ECAL Avalanche Photodiodes
}

\author{
N.Godinovic, I. Puljak, I. Soric, \\ Technical University of Split, Croatia \\ Z. Antunovic, M. Dzelalija \\ University of Split, Croatia \\ K. Deiters, Q. Ingram, D. Renker \\ Paul Scherrer Institute, Villigen, Switzerland \\ Y. Musienko 1 \\ Northeastern University, Boston, USA
}

\begin{abstract}
The photodetectors which will read out the scintillation light generated in the lead tungstate crystals in the barrel part of the CMS electromagnetic calorimeter are avalanche photodiodes (APDs). Scanning the APD's active area with a collimated light spot, the spatial uniformity of their quantum efficiency and gain has been measured at four different operating gains. Details of the APD surface structure are examined by scanning with a fine light spot. These details help to explain the difference between the bias voltage for a given gain when the full APD area is illuminated and when only the central part is illuminated.
\end{abstract}

1 on leave from INR (Moscow) 


\section{Introduction}

The design of the Compact Muon Solenoid (CMS) and the operating conditions at the Large Hadron Collider (LHC) require specially developed photodetectors to read out the lead tungstate $\left(\mathrm{PbWO}_{4}\right)$ crystals in the barrel part of the CMS electromagnetic calorimeter (ECAL) [1]. Avalanche photodiodes (APDs) were developed for this purpose by Hamamatsu Photonics in close collaboration with CMS. The APDs have an internal gain and an optimized area to cope with the modest light yield of the $\mathrm{PbWO}_{4}$ crystal; their properties are described elsewhere [3-7].

Until recently it was difficult to produce APDs with a sensitive area bigger than $1 \mathrm{~mm}^{2}$ due to the increasing probability of defects in large dimensions and the resulting low production yield. Technology improvements in the last decade have enabled the production of large area APDs [2]. Those which will be used in the CMS ECAL have an area of $5 \times 5 \mathrm{~mm}^{2}$.

Measurements of the photocurrent as a function of the position of a collimated DC light source across the APDs quantify the level of the spatial uniformity of the APD response. Any significant non-uniformity of the photodetector response with position will increase the effective excess noise factor of the diode. The measurement of the spatial uniformity also provides information about the quality of the production process.

In this paper measurements of the spatial uniformity of quantum efficiency and gains are reported in Section 3. Section 4 discusses how the illumination affects the measurement of the bias voltage for a given gain.

\section{The set-up for uniformity measurements}

The apparatus used to measure the APD uniformity is shown schematically in Figure 1. It consists of a

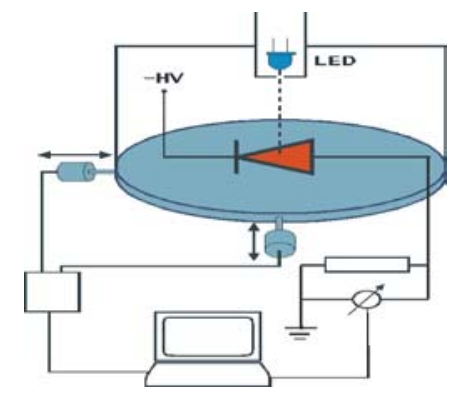

Figure 1. Schematic of the apparatus for uniformity measurements.

moveable table with stepping motors mounted on micrometers screws, which move the table along two perpendicular directions, each with a range of $10 \mathrm{~mm}$. Specially developed software written with Labview controls the stepping motors via a parallel port. LED light is transported to the APD faceplate by an optical fibre mounted on the moveable table. The APD is fixed and the collimated light spot of 200 microns diameter scans across its faceplate. The software allows the choice of different ways of scanning and variation of the step size as a multiple of $2.5 \mu \mathrm{m}$. A voltage source (ORTEC 451) was used for the APD bias. The photocurrent was determined by measuring the voltage drop across a $1 \mathrm{M} \Omega$ resistor $(0.1 \%$ precision $)$ in series with the APD, using 
a multimeter Keithley 2000. The multimeter and the temperature, measured by a PT 100 temperature probe (Pico Technology $0.1^{\circ} \mathrm{C}$ precision), were read out through two RS232 ports.

\section{APD uniformity at different gains}

The uniformity of the responses of a five APDs at different gains have been measured with this apparatus. An area of $8 \times 8 \mathrm{~mm}^{2}$ was scanned in a steps of 200 microns, giving in total 1600 measurement points for each APD. These measurements were carried out at a temperature of $25^{\circ} \mathrm{C}$, stabilized to $\pm 0.2{ }^{\circ} \mathrm{C}$. Repeated scans at the same gain but with different scanning rasters showed reproducibility at the level of $\pm 1 \%$. Variations at this level may be due to light source or residual temperature instabilities.

The uniformity measured at gain 1 is a measure of the spatial uniformity of the quantum efficiency. Figure 2 shows the illuminated current from the APD $\left(\mathrm{I}_{\mathrm{ph}}\right)$ at gain 1, measured with a 200 micron diameter light spot from a blue LED with maximum emission at $480 \mathrm{~nm}$. Applying a cut on the measured currents $\left(\mathrm{I}_{\mathrm{ph}}>0.75\right.$ $\mathrm{nA}$ ) selects the currents measured when the light spot was inside the APD photosensitive area. The variation of the current over the APD photosensitive area is shown on the right-hand plot in Figure 2, giving a spatial uniformity of the quantum efficiency at about $2 \%$, which is the level of the measurement's precision.
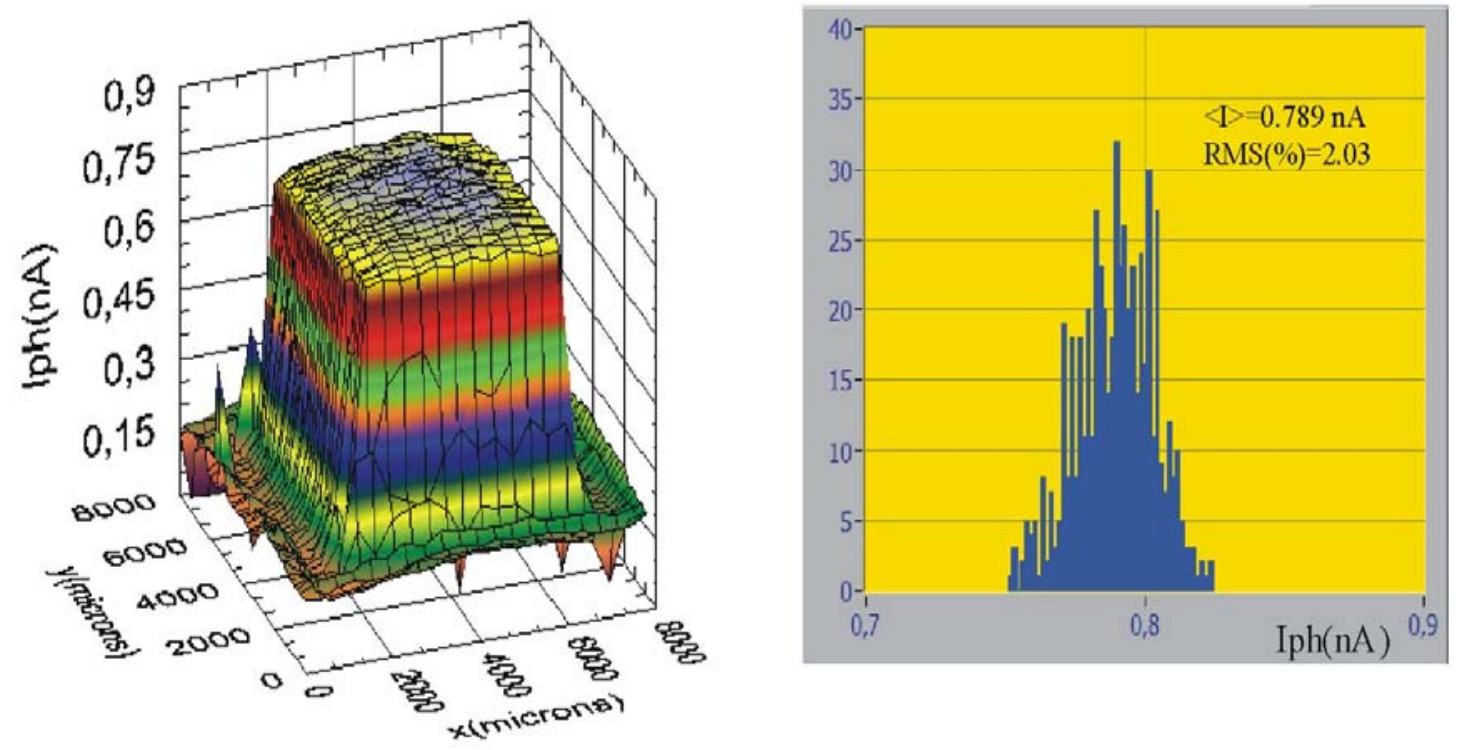

Figure 2. The current from an APD measured at gain 1 with an illumination spot of 200 microns diameter and scan steps of 200 microns. The plot on the right shows the distribution of the currents over the APD photosensitive area.

Figure 3 shows the uniformity of the APD measured at four different gains $(M=50, M=100, M=250$ and $\mathrm{M}=490$ ) with the same illumination and scan steps. Figure 4 shows the current distributions over the photosensitive area of the APD at the four gains, obtained by applying a cut on the measured currents $\left(\mathrm{I}_{\mathrm{ph}}>75\right.$ $\mathrm{nA}$ for gain 50, $\mathrm{I}_{\mathrm{ph}}>90 \mathrm{nA}$ for gain 100, $\mathrm{I}_{\mathrm{ph}}>350 \mathrm{nA}$ for gain 250 and $\mathrm{I}_{\mathrm{ph}}>700 \mathrm{nA}$ for gain 490). The mean value of the current and the r.m.s. of the distribution over the photosensitive area of $5 \times 5 \mathrm{~mm}^{2}$ are indicated in each plot. The r.m.s of the current distribution as a percentage of the mean photocurrent, summarised in Table 1, quantifies the level of the spatial non-uniformity of the gain. The r.m.s. non-uniformity measured with the 200 microns steps at gain 50 (the working gain of the APDs in the CMS ECAL) is $1.8 \%$, again comparable to the precision of the measurements. At a gain of 100 the non-uniformity is $2.1 \%$, also very good. At higher APD gains, $M=250$ and $M=490$, significant non-uniformities appear, of $5.8 \%$ and $7.8 \%$, respectively. 


\begin{tabular}{||c|c|c|c||}
\hline \hline GAIN (M) & $\bar{I}_{P h}(n A)$ & $\sigma_{\bar{I}_{p h}}(n A)$ & r.m.s. (\%) \\
\hline 50 & 81 & 1.5 & 1.8 \\
\hline 100 & 110 & 2.3 & 2.1 \\
\hline 250 & 438 & 25 & 5.8 \\
\hline 490 & 850 & 66 & 7.8 \\
\hline
\end{tabular}

Table 1. Average current over the photosensitive area of an APD, its standard deviation and percentage of nonuniformity, defined as the ratio of the two, at four different gains.
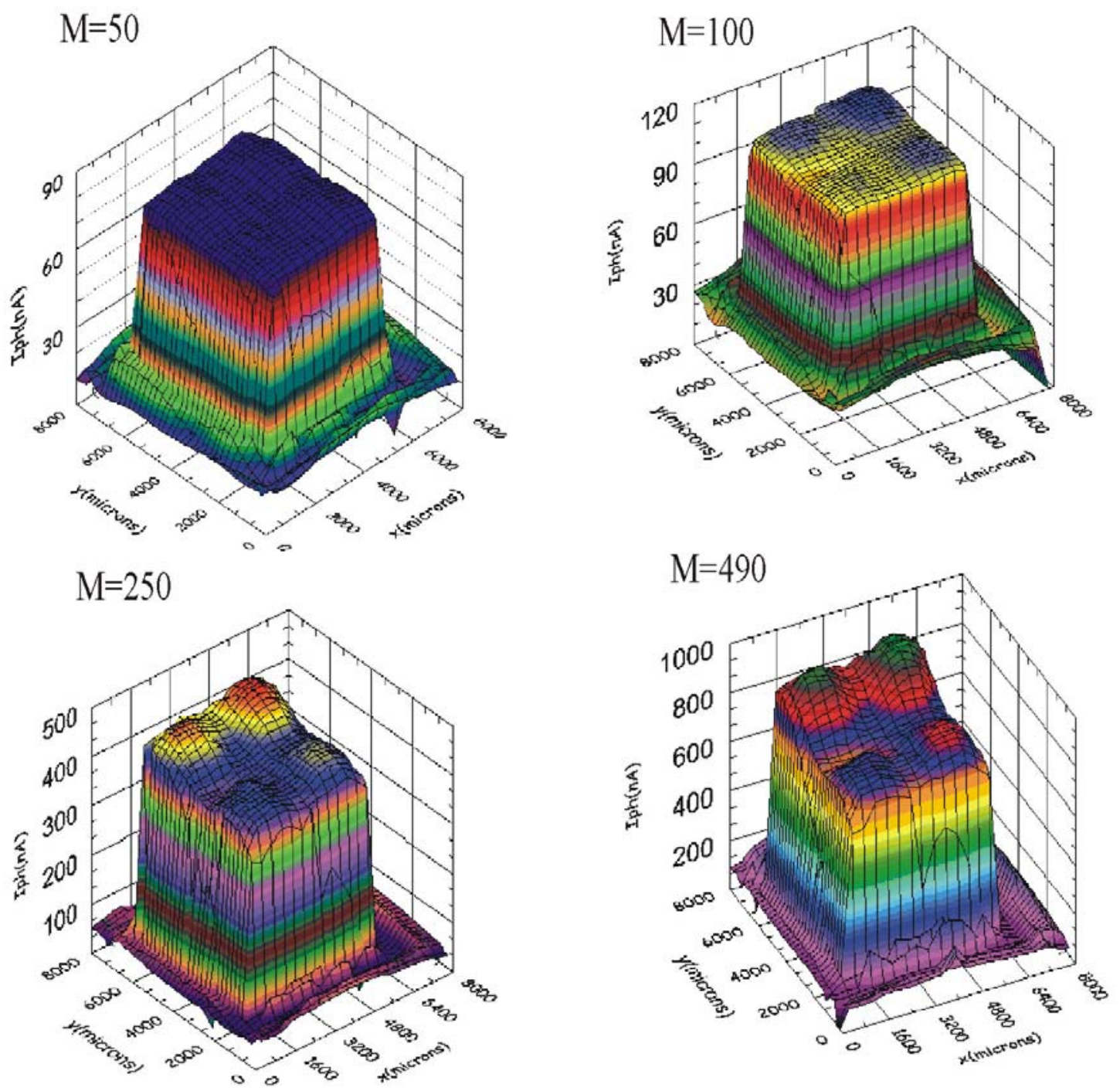

Figure 3. The current from an APD at four different gains measured with a blue LED (maximum emission at 480 $\mathrm{nm}$ ), a spot size of $200 \mu \mathrm{m}$ and scan steps of $200 \mu \mathrm{m}$. The currents do not scale with gain because the measurements were done with different light intensities. 

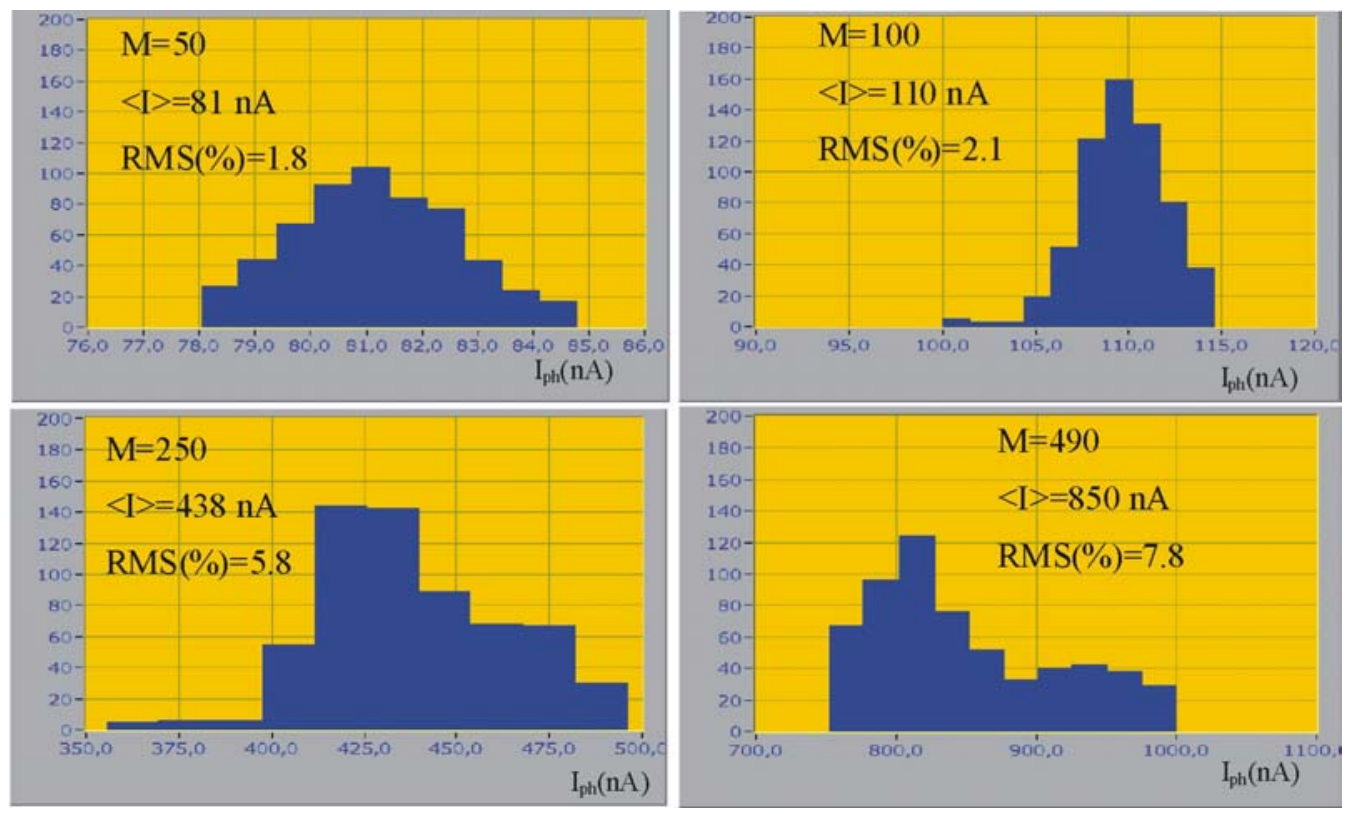

Figure 4. Distributions of the current over the photosensitive area of an APD shown as isometric plots in Figure 3.

The gain is determined by the internal electric field of the APD, which is a function of the applied bias voltage, the device structure and doping profile. Inhomogeneities in the electric field in the avalanche region will lead to non-uniformities of the gain. The excellent uniformity at gains 50 and 100 indicates a high level of control of the doping profile over the APD area, no significant defects in the crystal lattice and a proper design of the device structure. Imperfections in the device only become apparent at gains well above the gain $(\mathrm{M}=50)$ to be used in CMS.

\subsection{Discussion}

The noise property of avalanche multiplication processes is characterized by the effective excess noise factor $(\mathrm{F})$, defined by $\mathrm{F}=1+\left(\sigma_{\mathrm{M}} /<\mathrm{M}>\right)^{2},<\mathrm{M}>$ is the mean gain for single photoelectron and $\sigma_{\mathrm{M}}$ is the corresponding r.m.s. variation [1]. Generally the excess noise factor accounts for fluctuations due to the random nature of the avalanche process, but also for other variation of the gain. However the spatial non-uniformity of the gain can give a contribution to the value of $\mathrm{F}$ only if there is a non linear dependence of the excess noise factor on gain. The measured value of the excess noise factor for the CMS APD is F=2.1 \pm 0.2 at gain 50 and the observed linear rise in $\mathrm{F}$ with gain for gains bigger than 20 [4], imply a negligible contribution to F.

\section{The correlation between mean gain and the way of illumination}

Most of the APD surface outside of the active area is covered with aluminium. However, there are two small regions without aluminium, where the APD is light sensitive but with an unknown response. An understanding of the photo-response in these regions is relevant to the determination of the mean gain of the APDs. Figure 5 shows a photograph of the corner of an APD taken through a microscope. A light sensitive region starts 250 microns from the active region and is 90 microns wide. After a further 70 microns wide aluminised region a second light-sensitive region, the 100 microns wide groove, starts 410 microns from the active area. The edge of the APD also has a narrow exposed strip.

To understand the APD response in detail over the full surface, it was scanned with a fine light spot at two different gains, 1 and 50, with a scan step of 25 microns. Blue LED light was transported to the APD by a single mode fibre, 10 microns diameter to give a light spot of approximately 25 microns diameter. The measured current of an APD with gain 1 and 50 as a function of the spot position, are shown in Figure 6 over an area extending to the edge of the APD. Figure 7 shows the current at the two gains as a function of the spot position along a line traversing the APD. The figures for gain 1 show that the first light sensitive region is as sensitive to illumination as the active $5 \times 5 \mathrm{~mm}^{2}$ part of the APD, and that the exposed groove also has substantial sensitivity 
to light. In the figures for gain 50 on the other hand, these exposed regions at the edge of the diode are barely visible, indicating that there is very little or no amplification of the signal in these regions. Hence with full illumination of the diode there is some contribution to the average gain from the exposed edge regions at gain 1 , that is negligible when the APD is operated with a gain of 50 .

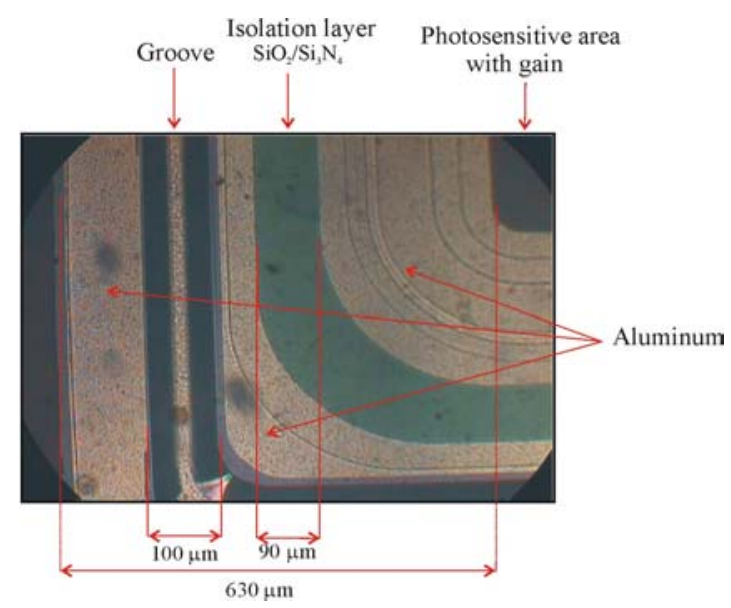

Figure 5. Photograph of the corner of an APD taken with a microscope
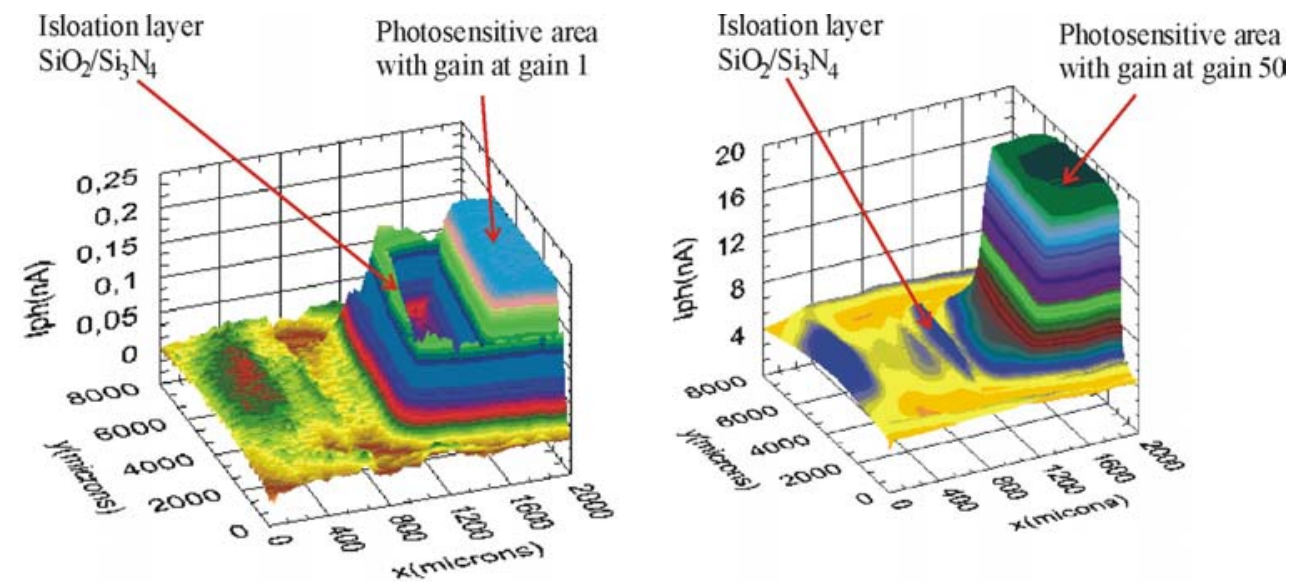

Figure 6. The current from an APD at gain 1 (left) and gain 50 (right) measured with a blue LED (peak emission at $480 \mathrm{~nm}$ ) with an illumination spot of 25 microns and scanning step of 25 microns.
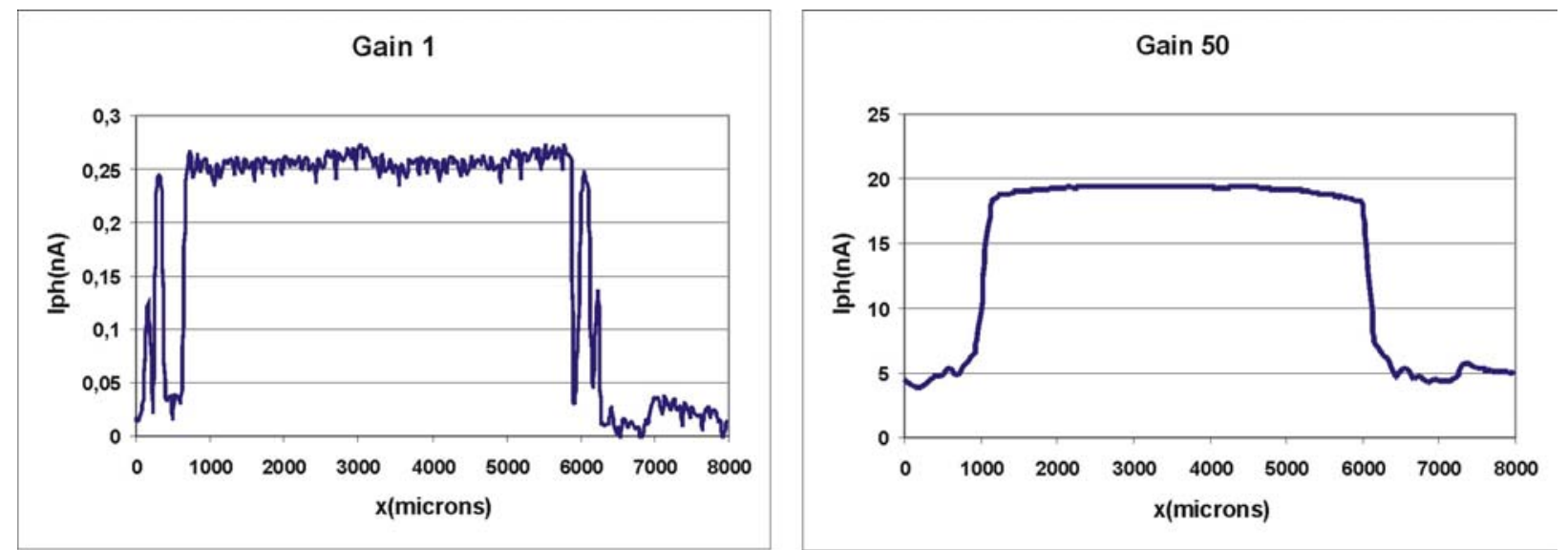

Figure 7. The measured current at gain 1 (left) and at gain 50 (right) as a function of the light spot position along a line traversing an APD. 
In the determination of the bias voltage for gain 50, we define the current for gain 1 as that observed with a low bias voltage, $20 \mathrm{~V}$. The voltage for gain 50 is then defined as that which gives 50 times the current observed at $20 \mathrm{~V}$. Since with full illumination the edge regions contribute to the observed current at gain 1 but negligibly at gain 50, the determination of the operating voltage at gain 50 gives different results depending on whether the edge regions are illuminated or not. A difference of about $3 \mathrm{~V}$ in the value of the operating voltage for the mean gain 50 was observed when the method of illumination was changed from full illumination to one with the edge regions covered by a mask. A simple estimation of gain difference for two methods of illumination at same operating voltage can be obtained calculating the effective increase in the photosensitive area which according to Figures 5 and 7 is about $10 \%$. Since the gain sensitivity to the bias voltage variations is $3.3 \% / \mathrm{V}$ at gain 50 [4], it follows that the expected operating voltage difference for gain 50 is $3 \mathrm{~V}$, in good agreement with the observed one.

\section{Conclusion}

The APDs have excellent spatial uniformity of the gain and of the quantum efficiency when operated at a gain of 50, as they will be used in the CMS ECAL detector. This precludes any potential contribution to the excess noise factor and indicates very good quality control during their production.

We have found a non-negligible sensitivity to light in the exposed edge regions of the diode, where there is little or no amplification compared to the operating gain of 50 . This sensitivity must be taken into account when determining the mean APD response.

\section{Reference}

[1] CMS Collaboration, The electromagnetic calorimeter project, Technical Design Report, CERN/LHC 97-33, December 1997.

[2] E. Lorenz et al, Nucl. Instrum. Meth. A344 (1994) 64.

[3] Q. Ingram et al, Avalanche Photodiode for CMS electromagnetic Calorimeter, Proceedings of International Europhysics Conference on HEP, Budapest 2001, hep2001/256.

[4] D. Renker, Nucl. Instrum. Meth. A486 (2002) 164.

[5] K. Deiters et al, Nucl. Instrum. Meth. A451 (2001) 574.

[6] A. Karar et al, Nucl. Instrum. Meth. A428 (1999) 413.

[7] T. Kirn et al, Nucl. Instrum. Meth. A387 (1997) 202. 